\title{
Transnationale Migration und die Dynamik von Rechten
}

\begin{abstract}
Welche Bedeutung hat die Staatsangehörigkeit noch in einer Welt zunehmender Migrationsbewegungen? Werden Migrantinnen und Migranten, solange sie nicht die Staatsangehörigkeit ihres Aufenthaltslandes erwerben (können), tendenziell zu Rechtlosen? Oder treten universelle Rechte an die Stelle von Staatsbürgerrechten? Die Spanne zwischen diesen beiden Extrempositionen - so das zentrale Argument dieses Aufsatzes wird von einer komplexen Anordnung unterschiedlicher Rechtsstellungen ausgefüllt, die als „bürgerrechtliche Schichtung“ bezeichnet werden. Jenseits des formellen Rechtsstatus verfügen Gruppen von Eingewanderten in unterschiedlichem Grade über „moralische Geltung", und zwischen ihrer formellen und ihrer informellen Stellung bestehen dynamische Wechselwirkungen. ${ }^{1}$
\end{abstract}

LYDIA MORRIS

\section{Einleitung}

Im vorliegenden Aufsatz schlage ich einen Ansatz vor, der dazu dienen kann, die Rechte von Migrantinnen und Migranten, die nicht die Staatsbürgerschaft des Zuwanderungslandes besitzen, zu verstehen und zu analysieren. Dabei argumentiere ich, dass die Spanne zwischen den beiden Extrempositionen - vollständige Verwehrung beziehungsweise uneingeschränkte Gewährung von Bürgerrechten von einer komplexen Anordnung unterschiedlicher Rechtsstellungen ausgefüllt wird, was ich im Folgenden als „bürgerrechliche Schichtung“ (civic stratification) bezeichne. Im Folgenden soll die Funktionsweise dieses Systems in Grundzügen dargestellt und erklärt werden; mein Aufsatz verfolgt also im Wesentlichen einen konzeptionellen Ansatz. Ein wichtiger Aspekt dieser Analyse ist der Zusammenhang zwischen formellem Rechtsstatus und informeller „moralischer" Geltung (standing) in der Gesellschaft. Um zu zeigen, wie die formelle Behandlung von Einwanderern die öffentliche Wahrnehmung beeinflussen kann, werde ich mich konkreter Beispiele aus dem Vereinigten Königreich bedienen; vermutlich spielen sich aber die beschriebenen Vorgänge auch anderswo ab.

Die Zeit an der Schwelle zum dritten Jahrtausend wurde als "the age of migration“ (Castles/Miller 1993) und als "the age of rights" (Bobbio 1995) bezeichnet; bislang jedoch blieb der Zusammenhang zwischen diesen beiden Charak- terisierungen ungeklärt. Die Tatsache der Anwesenheit von Eingewanderten ohne staatsbürgerliche Mitgliedschaft im Hoheitsgebiet der Nationalstaaten löste eine weitreichende Debatte über Art und Umfang der legitimen Ansprüche der Eingewanderten auf Rechte aus. Als Hannah Arendt (1979 [1948]) ihre berühmt gewordene Forderung nach dem „Recht, Rechte zu haben“ formulierte, reflektierte sie das problematische Verhältnis zwischen Staatsbürgerschaft und Menschenrechten, das insbesondere in der Position von Migrantinnen, Migranten und Asylsuchenden deutlich wird. Ungefähr zeitgleich mit der Formulierung der Allgemeinen Erklärung der Menschenrechte und im Rückblick auf die Menschen, die durch den Ersten Weltkrieg staatenlos geworden waren, konstatierte sie folgendes Paradox: „We became aware of the existence of a right to have rights and a right to belong to some kind of organised community only when millions of people had emerged who had lost and could not regain those rights." (Arendt 1979, S. 296f.)

Anders ausgedrückt, es macht keinen Sinn, von einem Rechtsanspruch zu reden, ohne eine anerkannte Beziehung zu einer Körperschaft, welche die Verpflichtung und Fähigkeit hat, diese jeweiligen Rechte zu achten und zu wahren. Auch heute noch werden Rechte in der Regel durch das nationalstaatliche System gewährleistet. Allerdings hat

1 Übersetzung aus dem Englischen von Lisa Eskuche. 
sich die Situation seit Arendts Beobachtung insofern etwas geändert, als inzwischen eine Fülle von Abkommen und Konventionen existiert, auf die sich grenzüberschreitend Wandernde und Asylanträge Stellende berufen können. Es ist jedoch nach wie vor der Nationalstaat, der diese Verträge ratifiziert, und es ist der Staat, der durch die Abkommen verpflichtet wird und die oberste Verwaltungszuständigkeit für die Umsetzung der jeweiligen Rechtsordnung behält. Die Frage, wie der rechtliche und soziale Status von Migrantinnen und Migranten in einer Position außerhalb nationaler Zugehörigkeit zu verstehen ist, ist noch keineswegs endgültig geklärt. Arendts Paradox beruhte auf der Tatsache, dass Menschen ohne Vollmitgliedschaft im nationalen Gemeinwesen via Staatsbürgerschaft keine Möglichkeit hatten, ihre „unveräußerlichen“ Menschenrechte einzufordern. Beiträge aus jüngerer Zeit hingegen kehren die Problematik um und stellen das Konzept der „Mitgliedschaft“ in Nationalverbänden vor dem Hintergrund der Existenz von NichtStaatsbürgern mit erheblichen Ansprüchen auf Rechte grundsätzlich infrage. In diese Richtung geht folgende Aussage: „A new and more universal concept of citizenship has unfolded in the post-war era, one whose organising and legitimating principles are based on universal personhood rather than national belonging." (Soysal 1994, S. 1)

Diese Ansicht ist jedoch nicht unumstritten. Arendts Argumentation findet ein einprägsames Echo in Benhabibs Beobachtung (2004, S. 2) aus jüngerer Zeit: „There is not only a tension but often an outright contradiction, between human rights declarations and states' sovereign claims to control their borders as well as to monitor the quality and quantity of admittees."

In der Praxis jedoch bleibt der Nationalstaat der vorrangige Vermittler des Zugangs selbst zu universellen Menschenrechten, und ihre Umsetzung hängt von ihm ab. Die akademische Diskussion über die Stellung und Bedeutung von Eingewanderten und Asylsuchenden auf nationalstaatlichem Gebiet hat zwei potenziell gegensätzliche Sichtweisen hervorgebracht (siehe Joppke 1999). Während eine Position den Fortbestand nationalstaatlicher Macht hervorhebt, die sich in seiner Kontrolle über die Einreise- und Aufenthaltsbedingungen in seinem Territorium manifestiert, und auf den Fortbestand der symbolischen und materiellen Bedeutung der nationalen Staatsbürgerschaft abstellt (siehe Brubaker 1989), sieht die andere Position die Präsenz von Eingewanderten mit legitimen Ansprüchen auf Rechte als Manifestation einer aufkeimenden „postnationalen“ Gesellschaft, in der nationale Staatsbürgerschaften verdrängt werden von Rechten, die an den Ort des gewöhnlichen Aufenthalts geknüpft sind (Soysal 1994). Dabei bildet die Europäische Union für Vertreter der postnationalen Sichtweise (z. B. Sassen 1998) einen gemeinsamen Bezugspunkt, da die EU eine multinationale Staatsbürgerschaft einzigartiger Prägung geschaffen hat, welche ein Recht auf Freizügigkeit innerhalb der Union und an die Freizügigkeit anschließende Rechte begründet. Allerdings privilegieren diese Rechte EU-Bürger gegenüber Angehörigen von Dritt- staaten; zudem ist unbestritten, dass sich die Freizügigkeit innerhalb der EU auf verschärfte Kontrollen an den Außengrenzen stützt - ein Umstand, der auch in den Bedingungen für den Unionsbeitritt zum Ausdruck kommt. Überdies steht ein weitgefasstes Verständnis universeller Grundrechte im Sinne einer Neubestimmung von Gesellschaft und gesellschaftlicher Zugehörigkeit in merkwürdigem Gegensatz zu der fast täglich zu beobachtenden Verteidigung nationaler Ressourcen und zu den aggressiven Zuwanderungskontrollen gegenüber Einwanderungswilligen, die sich abmühen, die EU-Grenzen zu überwinden, und zu der Situation, dass abgelehnte Asylbewerber in Gefahrensituationen zurückgeschickt werden.

Beide Sichtweisen, nationale Abschottung beziehungsweise postnationale Expansion von Bürgerrechten, lassen sich bis zu einem gewissen Grade belegen, doch für sich genommen wird jede der beiden Positionen den Erfahrungen der Migrantinnen und Migranten und den damit verbundenen politischen Reaktionen nur teilweise gerecht. Obwohl Wissenschaftler beider Lager Widersprüche und Spannungsverhältnisse einräumen, wird dieser Problematik erst seit relativ kurzer Zeit vertiefend nachgegangen. Auf der Suche nach einem konzeptionellen Weg, mit diesem Problem umzugehen, ohne eine der beiden Positionen zu übertreiben oder zu verneinen, fand ich es sinnvoll, einander widerstreitende Zwänge auszumachen und zu untersuchen, die auf der Ebene der Union und ihrer Mitgliedstaaten wirksam sind (Morris 2003). So wirken Anerkennung und Achtung von Grund- und Menschenrechten migrationsfördernd, etwa in Gestalt der Familiennachzugs- und Asylpolitik; Gleiches gilt für den anhaltenden Arbeitskräftebedarf am oberen und unteren Ende der Qualifikationsskala. Migrationshemmende Wirkung entfalten hingegen Beschränkungen des Anspruchs auf Sozialleistungen und des Arbeitsmarktzugangs auf einzelstaatlicher und EUEbene sowie die Einschränkung von Menschenrechten durch an sie geknüpfte Bedingungen oder Abschreckungsmittel. Folglich muss die Analyse sich darauf konzentrieren, wie sich solche Spannungen in Politik und Praxis umsetzen und wie ein Mittelweg zwischen den beiden scheinbar gegensätzlichen Positionen - postnationale Ausweitung von Rechten bzw. nationale Abschottung - aussehen könnte.

\section{Bürgerrechtliche Schichtung und die Ausgestaltung verschiedener Rechts- stellungen von Eingewanderten}

\subsection{Diskussion unterschiedlicher Ansätze}

Dass die Bedingungen für Anwesenheit und dauerhaften Aufenthalt auf staatlichem Hoheitsgebiet durch unterschiedliche Rechtsstellungen reguliert sind, ist schon lange unum- 
stritten. Hammars (1990) dreigliedrige Unterscheidung zwischen Staatsbürgern (citizens), Wohnbürgern (denizens) und Fremden (aliens) hat durch die EU-Bürgerschaft eine weitere Differenzierung erfahren. Die Realität gestaltet sich allerdings bedeutend komplizierter als Hammars Modell unterstellt und wird überdies immer komplexer - im Bereich der Einwanderung wird die Hierarchie der Rechtsstellungen um immer neue Abstufungen ergänzt, von denen jede mit einer jeweils anderen Palette von Rechten verknüpft ist. Dieses Phänomen lässt sich als System bürgerrechtlicher Schichtung (civic stratification) verstehen, ein Begriff, der durch David Lockwood (1996) eingeführt wurde, um eine Struktur gesellschaftlicher Unterschiede zu beschreiben, die aus der Gewährung oder Verwehrung von Rechten durch den Staat erwachsen ist. Als Lockwood diesen Begriff prägte, ging es ihm in erster Linie um Ungleichheiten, die der internen Funktionsweise von an Staatsbürgerschaft geknüpften Rechten entspringen; in meinen eigenen Untersuchungen übertrage ich Lockwoods Konzept auf die Analyse von Einwanderungspolitik. Das ermöglicht es uns, bei der Betrachtung von Rechten und ihrer Gewährung und Nutzung über die Staatsbürgerschaft hinauszudenken, ohne dabei die Frage der tatsächlichen Umsetzung universeller Grundrechte aus dem Auge zu verlieren. Sie steckt zudem den Rahmen ab für eine Diskussion des Rechtesystems als einem dynamischen sozialen Prozess, der genauere Aufmerksamkeit verdient.

Lockwood identifiziert in seiner Darstellung bürgerrechtlicher Schichtung zwei Achsen der Ungleichheit - Gewährung oder Nichtgewährung von Rechten sowie Besitz moralischer und materieller Ressourcen. In der ersten Dimension geht es um den formellen Rechtsanspruch, in der zweiten um Diskriminierung oder um Prestigefaktoren, die die tatsächlichen Möglichkeiten der Inanspruchnahme von Rechten beeinflussen; diese zweite Achse erweitert die Analyse somit um einen informellen Aspekt. In Bezug auf Migration findet sich auf der formellen Ebene eine Vielzahl von Rechtsstellungen, die innerhalb der EU sowohl nationalem als auch EU-Recht unterliegen und im Kontext von Verpflichtungen aus den allgemeinen Menschenrechten umgesetzt werden müssen. Diese verschiedenen Rechtsstellungen von Eingewanderten werden durch den Grund der Einreise bestimmt und sind an unterschiedliche Bedingungen oder Einschränkungen gebunden. Rechte lassen sich folglich als herrschaftliches Steuerungsinstrument auffassen. In der Tat ist die Gewährung von Rechten oftmals mit Möglichkeiten behördlicher Überwachung verknüpft, sodass zwischen Rechten und Kontrolle ein relativ enger Zusammenhang besteht. Die hier im Wesentlichen infrage stehenden Rechte umfassen Sozialleistungen, Arbeit, Aufenthalt, Familiennachzug sowie Asyl- oder schwächere Schutzrechte; sie können auf verschiedene Weise mit Steuerungszwecken verknüpft werden.

Es ist gang und gäbe, Sozialleistungen zu Beginn des Aufenthalts zu verweigern; zudem ist die Leistungsgewährung unter Umständen an eine Überprüfung des Einwan- derungsstatus gekoppelt (siehe Morissens/Sainsbury 2005). Die frühere Inanspruchnahme von Sozialleistungen kann wiederum bei Antrag auf unbefristeten Aufenthalt eine Rolle spielen. Bestimmte Beschäftigtengruppen können Vorzugsregelungen in Bezug auf Arbeits- und Aufenthaltsbewilligung genießen. Andererseits kann der eingeschränkte Zugang zu Rechten als Abschreckungsmittel eingesetzt werden - man denke nur an das Arbeitsverbot für Asylsuchende oder die Herabstufung der Sozialleistungsansprüche dieser Gruppe. Durch Vorbedingungen im Bereich des Familiennachzugs, etwa hinsichtlich der Unterbringung und des Unterhalts eines zureisenden Ehepartners, lässt sich die Zuwanderung begrenzen; bestimmten Gruppen Wanderungswilliger können Nachzugsrechte verweigert werden. Schließlich lassen sich Unterstützungsleistungen für Asylsuchende an Meldepflichten knüpfen. Überhaupt ist oftmals das Gefüge der Zivilgesellschaft von der Mikro- bis zur Makroebene von verschiedenen Formen regulatorischer Aufsicht durchdrungen, die sich im Rahmen dessen, was Foucault (1991) als Gouvernementalitätsprozess bezeichnete, entsprechender Kontrolltechniken und -verfahren bedienen.

\subsection{Bürgerrechtliche Schichtung in der EU}

Natürlich setzen innerhalb der EU mit der EU-Bürgerschaft verbundene Rechte einige der genannten Einschränkungen durch den Freizügigkeitsgrundsatz außer Kraft. Dennoch haben sich auch hier Spielräume für Differenzierungen ergeben - beispielsweise der nur schrittweise Zugang zu Arbeitsmärkten und sozialen Rechten für Bürger der neueren Mitgliedsländer. Überdies erweitert das EU-Recht per se die auf nationalstaatlicher Ebene bestehende immigrationsrechtliche Abstufung um eine zusätzliche Dimension. Und wenngleich menschenrechtliche Verpflichtungen nunmehr im Vertrag von Lissabon festgeschrieben wurden, ist deren Umsetzung durchaus mit Unterscheidungen der beschriebenen Art vereinbar. Zum Beispiel enthält die Europäische Menschenrechtskonvention eine eigene Rangordnung absoluter, begrenzter und qualifizierter Rechte, wobei unter "qualifiziert" die Abhängigkeit von gewissen Bedingungen zu verstehen ist. Ein Beispiel hierfür ist eine Koppelung des Rechtes auf Familiennachzug an das wirtschaftliche Wohl des Aufnahmelandes. Zudem sind selbst absolute Rechte oft eine Frage der Auslegung (siehe zum Beispiel Morris 2010) und werden unter Umständen durch Umsetzungsmängel ausgehöhlt; so erschwert beispielsweise die Kombination von Visavorschriften mit der Möglichkeit, Sanktionen gegen Personenbeförderungsunternehmen zu verhängen, die Inanspruchnahme des Rechts auf Asylbeantragung (Cruz 1995).

Die Auseinandersetzung mit dem Rechtsgefüge der einzelnen EU-Mitgliedstaaten setzt also ein Verständnis der höchst unterschiedlichen Funktionsweisen und Wechselwirkungen von nationalem und europäischem Recht sowie völkerrechtlicher Konventionen voraus, sowohl in Bezug auf die jeweiligen formellen Inhalte als auch 
auf ihre praktische Anwendung. Die Bandbreite der möglichen - und jeweils mit einer unterschiedlichen Palette von Rechten ausgestatteten - Rechtsstellungen erstreckt sich, grob gesagt, vom

- Staatsbürger (kraft Geburt oder Einbürgerung) über

- Arbeitnehmer aus EU-Ländern und

- deren Familienangehörige

- Nicht-Arbeitnehmer aus EU-Mitgliedsländern

- Arbeitnehmer mit unbefristeter Aufenthaltsgenehmigung aus Drittländern und

- deren Familienangehörige

- Arbeitnehmer aus Drittländern mit befristeter Arbeitsgenehmigung

- Zeitarbeitnehmer mit befristeter Aufenthaltserlaubnis

- anerkannte Flüchtlinge und deren Familienangehörige

- Personen, die aus humanitären Gründen Flüchtlingsschutz genießen

- noch nicht anerkannte sowie abgelehnte, aber nicht ausreisefähige Asylsuchende bis hin zu

- Eingewanderten ohne Ausweispapiere.

Die zahlenmäßige Verteilung dieser verschiedenen Rechtsstellungen dürfte aufgrund der Unterschiede zwischen den einzelstaatlichen Ordnungen von Land zu Land anders ausfallen. Zwar besteht auf EU-Ebene eine Bewegung hin zu einer Harmonisierung, doch im Wesentlichen gibt das EURecht lediglich Mindestanforderungen vor, die einzelstaatliche Abweichungen weiterhin ermöglichen. So ist zum Beispiel in Deutschland eine Diskussion entbrannt über die Sozialleistungsansprüche von Arbeitnehmerinnen und Arbeitnehmern, die ihr Freizügigkeitsrecht ausüben, ${ }^{2}$ während im Vereinigten Königreich der Umgang mit dieser Gruppe bereits kürzlich geändert wurde (Guardian 2014).

Jenseits der Anforderungen des Menschenrechts unterliegt der Umgang mit Drittstaatsangehörigen unterschiedlichen Kombinationen gemeinschaftsrechtlicher und einzelstaatlicher Einwanderungsvorschriften. Bezüglich der Freizügigkeit zwischen Mitgliedstaaten, zum Beispiel, gilt für Drittstaatsangehörige mit Wohnsitz in einem EU-Land, Inhaberinnen und Inhaber der Blauen Karte EU sowie Forscherinnen und Forscher und schließlich entsandte Arbeitskräfte eine EU-Richtlinie (siehe European Migration Network 2013), die jedoch dem Ermessen der Einzelstaaten einen erheblichen Spielraum lässt. Die 2009 eingeführte Blaue Karte erweitert die Rechte Hochqualifizierter im Vergleich zu anderen Drittstaatsangehörigen, stellt die Inhaberinnen und Inhaber jedoch nicht den EU-Bürgern gleich. Sie sollte für hochqualifizierte Drittstaatsangehörige Hindernisse der Arbeitsmarktmobilität zwischen EU-Mitgliedsländern ausräumen; allerdings schlossen sich Dänemark, Großbritannien und Irland dem nicht an. Andere Gruppen - etwa Beschäftigte mit befristeter Arbeitserlaubnis, Grenzgängerinnen und -gänger sowie Saisonarbeitskräfte - fallen nicht unter die EU-Richtlinie, während die Ersteinreise in ein EU-Mitgliedsland nationalem Recht unterliegt. In diesem Bereich bestehen weiter zulässige Un- terschiede; in Deutschland beispielsweise ist das Recht deutscher Bürger auf Familiennachzug lediglich an den Nachweis von Sprachkenntnissen gebunden, während im Vereinigten Königreich für Briten gleiche Anforderungen gelten wie für aufenthaltsberechtigte Nicht-Staatsbürger, nämlich die nachgewiesene Fähigkeit, den einreisenden Ehepartner angemessen unterbringen und unterhalten zu können.

\subsection{Dynamik bürgerrechtlicher Schichtungen}

Trotz erheblicher Schritte in Richtung Harmonisierung zwischen den Mitgliedstaaten bestehen zudem historisch gewachsene Unterschiede, die das Wesen und die Ausgestaltung der jeweiligen Einwanderungsregime nach wie vor beeinflussen. Zur Veranschaulichung ein kurzer Vergleich dreier kontrastierender Länder, mit denen ich mich befasst habe (Morris 2002): In Großbritannien hatte das Zuwanderungsrecht für Arbeitnehmer auf Basis der Zugehörigkeit zum Vereinigten Königreich und dessen Kolonien (Citizenship of the United Kingdom and Colonies) zur Folge, dass diese Migrantinnen und Migranten in der Nachkriegszeit automatisch uneingeschränkte Staatsbürgerrechte genossen. Den Angehörigen des New Commonwealth dagegen wurde im Zuge des Einwanderungsgesetzes von 1971 und der Einführung des "patriality requirements“ - das heißt der Voraussetzung einer „engen Verbindung“ zu Großbritannien kraft Geburt oder Abstammung - das Aufenthaltsrecht aberkannt. Im Kontrast dazu hatte Deutschland ein restriktiveres, wenn auch inzwischen etwas gelockertes Staatsbürgerschaftsregime, daneben Sonderregelungen für die Anwerbung von Gastarbeitern, und ist, im Gegensatz zu Großbritannien, weiterhin offiziell gegen eine doppelte Staatsbürgerschaft, auch wenn es sie unter bestimmten Bedingungen zulässt. Italien kann im Vergleich zu den beiden vorgenannten Ländern auf eine deutlich jüngere Geschichte einer zum großen Teil ohne die erforderlichen Papiere erfolgten Einwanderung zurückblicken - Eingewanderte, die zunächst im informellen Sektor beschäftigt waren, haben durch Regularisierung ihres Status ein Aufenthaltsrecht erworben. Ermöglicht wurde dies durch eine Reihe von Amnestie-Maßnahmen (sanatoria), die unter bestimmten Voraussetzungen - insbesondere dem Bestehen eines offiziellen Arbeitsverhältnisses - einen Übergang vom „papierlosen“ zum legalisierten Status eröffnen. Wiederum anders wird sich die Situation in den Ländern Osteuropas darstellen, wo das Migrationsgeschehen sowohl von Abwanderung als auch von sehr jungen Einwanderungsbewegungen und einem Zustrom Asylsuchender gekennzeichnet ist.

Großbritannien hat sich allmählich von seinen traditionellen Verbindungen zum Commonwealth gelöst und hat ein komplexeres System von Rechtsstellungen für

2 http://eurorights.net/euro-rights-blog-germanys. 
Eingewanderte, umfangreichere Vorbedingungen für die Einbürgerung und ein stärker abgestuftes System von Arbeitsgenehmigungen eingeführt. Deutschland hat sich unterdessen von einem hochbürokratischen, streng abgestuften Statussystem und einem exklusiven Staatsbürgerschaftsmodell verabschiedet und beides etwas einfacher und offener gestaltet. Damit entwickeln sich die beiden Länder in mancher Hinsicht konvergent. In Italien wurden die Kontrollen inzwischen ebenfalls verschärft und die Formalisierung vorangetrieben, doch sind die Einreise ohne Papiere und irreguläre Arbeitsmarktpraktiken nach wie vor ein wichtiges Merkmal des dortigen Systems. Trotz einer gewissen Konvergenz zeigt das Bild der bürgerrechtlichen Schichtung in den drei Ländern also spezifische Ausprägungen, die auf historische Unterschiede und deren unvermeidliche Wechselwirkungen mit der Rechtsordnung zurückgehen. Interessante Fragen ergeben sich dabei im Hinblick auf Veränderungen des Systems als solchem, dem Raum für Auf- oder Abstieg in der Hierarchie der Rechtsstellungen sowie bezüglich der Frage, inwieweit sich die bürgerrechtliche Schichtung mit sonstigen Unterschieden deckt, insbesondere im Hinblick auf nationale Herkunft, Hautfarbe ( race) oder ethnische Zugehörigkeit. Die Ergebnisse werden sicherlich von Land zu Land unterschiedlich ausfallen; allgemein besteht aber die Tendenz, dass die am wenigsten vorteilhaften Positionen von außereuropäischen Migrantinnen und Migranten nicht-weißer Hautfarbe eingenommen werden; andererseits können formelle Statusunterschiede und ungleicher Zugang zu Rechten durchaus auch quer zu einzelnen nationalen oder ethnischen Einteilungen liegen.

\subsection{Gender-Spezifik der Schichtungen}

Darüber hinaus kann man danach fragen, inwieweit rechtliche Stellung und persönliche Erfahrungen mit dem System genderspezifisch sind. Hier müssen wir auf die besonderen weiblichen Migrationsmuster und ihre Auswirkungen auf die persönliche Erfahrung und speziell auf den Zugang zu Rechten achten. In Bereichen wie Haushaltshilfe, Pflegearbeit, Sexarbeit und Ehepartnerzuzug, in denen die spezifische Zuordnung der Frau zur Privatsphäre zum Tragen kommt, sind Migrantinnen überproportional vertreten (Kofman et al. 2000). In Privathaushalten beschäftigte Haushaltshilfen und Pflegekräfte erleben mitunter Einschränkungen ihrer Privatsphäre und können ihre Arbeitnehmerinnenrechte oft nur mit Schwierigkeiten geltend machen. Gleichzeitig kann es sein, dass diese Migrantinnen auf andauernd gute Beziehungen zu ihrem Arbeitgeber angewiesen sind, um sich weiter im Land aufhalten zu können, sodass ihr Zugang zu Rechten über den jeweiligen Haushaltsvorstand vermittelt sein kann. In einer ähnlich abhängigen Position befinden sich nachziehende Ehepartnerinnen und -partner in den ersten Jahren ihres Aufenthalts; ihre Aufenthaltsberechtigung ist an den Fortbestand der ehelichen Beziehung geknüpft. Extremer ist die Situation papier- loser Sexarbeiterinnen, die unter Umständen quasi in Gefangenschaft gehalten werden und denen jeglicher öffentlicher Status verweigert wird. Es ist zudem allgemein anerkannt, dass Frauen hinsichtlich ihrer Aussichten auf Anerkennung durch das Asylsystem auf eine Weise benachteiligt sind (Crawley 1997), die einem Defizit in der Aussicht auf Nutzung dieses Rechts gleichkommt.

\section{Die informelle Dimension bürger- rechtlicher Schichtung}

Bis hierhin hat sich meine Erörterung auf das konzentriert, was ich als die formelle Dimension von Lockwoods Modell bezeichne, d. h. auf die abgestufte Gewährung von Rechten; dies schließt jedoch nicht aus, dass in vielen Situationen auch informelle Faktoren zum Tragen kommen. Daher soll im Folgenden die Bedeutung der informellen Dimension näher betrachtet werden, die sich mit den Begriffen Verstärkung (gain) und Mangel (deficit) beschreiben lässt. Ihre Triebfeder ist das, was man im weiteren Sinne als gesellschaftliche Geltung (standing in society) bezeichnen könnte, und dieses wird bestimmt durch Faktoren, die mit Reichtum, Einfluss und Prestige zusammenhängen. Lockwood (1996, S. 536) beschreibt diese Dimension wie folgt: „advantages conferred by social standing and social networks, command of information, and general know-how, including the ability to attain one's ends through the activation of shared moral sentiment[..].“

\subsection{Wechselwirkungen zwischen formeller und informeller Schichtung}

Während sich die zuvor beschriebene formelle Dimension der bürgerrechtlichen Schichtung in unterschiedlichen Rechtsstellungen ausdrückt, zeigt sich die informelle Seite, wo immer ein Gewinn an Prestige zu einer Vorzugsbehandlung führt, oder umgekehrt in indirekten Hindernissen, ein formal gegebenes Recht zu beanspruchen bzw. in Stigmatisierungen, die es unmöglich machen, Rechte in vollem Umfang wahrzunehmen. Beispiele für indirekte Hindernisse sind der Zwang zum Nachweis der Fähigkeit zur Unterbringung und zum Unterhalt nachziehender Familienangehöriger (der aufgrund der Benachteiligung am Arbeitsmarkt schwer zu erbringen sein mag) und das Misstrauen, das Wanderarbeitskräften entgegenschlägt, das noch verstärkt werden kann durch die Bedrohung der Arbeitgeber „papierloser“ Migrantinnen und Migranten mit Sanktionen. Ein Beispiel für eine Stigmatisierung, die Eingewanderte davon abhalten kann, Rechte in Anspruch zu nehmen, ist die negative Befrachtung der Bezeichnung "Asylant “ (oder gar „illegale Asylanten“) im Sinne der zunehmend verbreiteten Meinung, die meisten Asylsuchenden seien „Scheinasylanten“. 
Von besonderem Interesse ist jedoch der Umstand, dass der informelle Aspekt bürgerrechtlicher Schichtung das Modell um eine dynamische Komponente bereichert. Nach Lockwood kommen dem Besitz oder Zugewinn moralischer Ressourcen besondere Bedeutungen zu als Grundlage für die Ausweitung von Rechtsansprüchen. Lockwood legt bei der Erörterung dieser Möglichkeit den Schwerpunkt auf die „innere Logik“ der Staatsbürgerschaft, die darauf angelegt sei, ihre Grenzen zu überwinden und über das jeweils Erreichte hinausgehende Erwartungen zu schaffen; das Gleiche ließe sich für Rechte im allgemeinen Sinne sagen. Bei der Untersuchung dieses expansiven Potenzials bezieht sich Lockwood auf die Rolle der (von ihm selbst so benannten) Bürgerrechtsaktivisten (civic activists) bei der Mobilisierung der Öffentlichkeit für eine umfassendere Ausstattung mit Rechten, die sich nicht zuletzt in einer zunehmenden Anrufung der Gerichte äußert. Tatsächlich können wir eine Verbindung zwischen dieser Möglichkeit und Alexanders (2006) Beobachtung herstellen, dass das Recht nicht nur trennende und ausschließende Wirkung besitze, sondern auch die Möglichkeit der zivilgesellschaftlichen Korrektur (civil repair) biete. Die Konsolidierung allgemeiner Menschenrechte und deren Aufnahme in die nationalen Rechtsordnungen ist ein Schwerpunkt solcher Aktivitäten gewesen, besonders in Ländern, die Menschenrechtsnormen in nationales Recht umgesetzt haben und in denen deshalb entsprechende Rechtsmittel eingelegt werden können. Selbstverständlich lässt sich der Expansionsgedanke aber nicht nur auf ganze Rechtsordnungen anwenden, sondern ebenso auf die Erfahrungen einzelner Gruppen innerhalb eines Regimes, die - möglicherweise mit Unterstützung von Bürgerrechtsaktivisten - gesellschaftliche Geltung oder „moralische Ressourcen“ hinzugewinnen (dazu z. B. Morris 2010) und dadurch ihre formelle Stellung verbessern. Die Einführung der Blauen Karte EU für hochqualifizierte Drittstaatsangehörige ist ein mögliches Beispiel, ein weiteres wäre die immigrationsrechtliche Anerkennung gleichgeschlechtlicher Beziehungen zu Zwecken des Familiennachzugs. Kreative Expansion kam auch in verschiedenen Auslegungen der Genfer Flüchtlingskonvention hinsichtlich der Anerkennung von „Verfolgung wegen Zugehörigkeit zu einer bestimmten sozialen Gruppe“ als Asylgrund zum Ausdruck (siehe zum Beispiel die Entscheidung des Appellationsausschusses des Britischen Oberhauses in den Fällen zweier weiblicher Flüchtlinge, aus Iran und aus Sierra Leone). ${ }^{3}$

\subsection{Bürgerrechtliche Abstiegsdynamiken}

In Lockwoods Schema steht Mangel für das Gegenteil von Verstärkung, und obwohl Lockwood den Begriff in Bezug auf die Erfahrungen bestimmter Gruppen oder Individuen beim Zugang zu einem bestimmten Recht verwendet, lässt sich seine dynamische Funktion innerhalb einer ganzen Rechtsordnung weiter ausdifferenzieren. Im Zusammenhang mit Gender wurde oben aufgezeigt, dass die Zuordnung von Frauen zur häuslichen Sphäre sich in einen Man- gel beim Zugang von Rechten in der öffentlichen Sphäre umsetzen kann; ich habe aber auch andere Beispiele zitiert, die eine indirekte Wirkung bestimmter Kontrollpraktiken belegen. Die Bedeutung moralischer Ressourcen verdient an dieser Stelle besondere Aufmerksamkeit. Ein Mangel der beschriebenen Art liegt vor, wenn bereits die Gewährung eines Rechtes die Entstehung dessen bewirkt, was Lockwood (1996, S. 538) als „an incipient status group of a negatively privileged kind“ bezeichnet. Er erwartet solche Entwicklungen im Zusammenhang mit Konstellationen sozialer Abhängigkeit, wie im Falle von Sozialleistungsbezug. Ein Beispiel hierfür wären Asylsuchende, ein anderes die jüngste Panik in Großbritannien wegen EU-Migrantinnen und -Migranten aus Rumänien und Bulgarien, die angeblich durch das Sozialleistungssystems angezogen werden (Guardian 2013a). Die Wahrnehmung dieser Gruppe in der Öffentlichkeit hat zur Folge, dass es ihr aufgrund der unterstellten Beweggründe für ihre Einwanderung in Lockwoods Begriffen an moralischem Gewicht (moral leverage) mangelt; allerdings hängt die geringe Wertschätzung dieser Eingewanderten wohl auch mit kulturellen oder rassistischen Vorurteilen zusammen.

Während nach Lockwoods Konzept Verstärkungen der bürgerrechtlichen Stellung (civic gain) an einem Punkt das Potenzial besitzen, den Anstoß für die Verbesserungen der Stellung im gesamten Rechtssystem zu geben, wird die Mangelsituationen von ihm nicht entsprechend erörtert - vermutlich, weil Lockwoods Diskussionsrahmen auf zwei Gegensätzen aufbaut und keine dritte Möglichkeit vorsieht. Aus der Kombination der beiden von ihm postulierten "Achsen der Ungleichheit" leitet Lockwood vier Grundtypen bürgerrechtlicher Schichtung ab, bürgerrechtliche Exklusion und Expansion, Verstärkung und Mangel (civic exclusion und civic expansion, civic gain und civic deficit), also zwei Gegensatzpaare. Das Gegenteil von Exklusion ist jedoch Inklusion (nicht Expansion), womit Raum für die Erwägung eines dritten Gegensatzpaares, Expansion und Kontraktion, gegeben wäre, um den veränderlichen Charakter eines gesamten Rechtssystems oder eines bestimmten Bereichs in einem solchen System zu beschreiben. Anders gesagt: Wenn Rechte expandieren können, können sie sicher auch kontrahieren - und damit kommen wir zur Betrachtung des Verhältnisses zwischen den formellen und den informellen Komponenten bürgerrechtlicher Schichtung in Bezug sowohl auf öffentliche Politik als auch öffentliche Meinung. Verstärkte Aufmerksamkeit gebührt insbesondere der Dynamik, durch die einzelne Gruppen innerhalb einer Gesellschaft ihr Prestige erhöhen bzw. an Geltung einbüßen können, ebenso dem Verhältnis zwischen solchen Prozessen und dem politischen und öffentlichen Meinungsklima.

3 UK House of Lords [2006] UKHL 46, Secretary of State for the Home Department v. K. and Fornah, http://www. publications.parliament.uk/pa/ld200506/ldjudgmt/ jd061018/sshd-1.htm. 


\section{Die soziale und politische Dynamik gesellschaftlicher Geltung}

Kombiniert man die formellen und informellen Aspekte bürgerrechtlicher Schichtung miteinander und überträgt die resultierende Dynamik auf die Analyse der Zuwanderungsgesetze und die Herausbildung der verschiedenen Rechtsstellungen von Eingewanderten, so ergibt sich eine Reihe interessanter Einsichten. Es lässt sich zum Beispiel erkennen, dass die Herausbildung verschiedener Rechtsstellungen von Eingewanderten und die damit verbundene Möglichkeit eines engen Zusammenhangs zwischen Rechten und Kontrolle den Schluss nahelegen, dass der gesamte Komplex bürgerrechtlicher Schichtung vom Staat zu eigenen politischen Zwecken manipuliert werden kann. Trotz gewisser Einschränkungen durch Gemeinschaftsrecht und Menschenrechtsverpflichtungen stellt die Kontrolle über die Einreise in das Staatsgebiet ein heftig verteidigtes Privileg der Nationalstaaten dar, wenn auch - dank der Freizügigkeitsregelungen innerhalb der EU - in leicht modifizierter Form. Die Erweiterung von Rechten kann (wie im Falle der Blauen Karte) eingesetzt werden, um erwünschte Kategorien von Wanderungswilligen anzulocken, während die Verweigerung oder nur bedingte Gewährung von Rechten zur Abschreckung genutzt werden können. Derartige Manöver können auf die informelle Dimension der Rechte durchschlagen und das öffentliche Bild einzelner Migrantengruppen bestimmen; öffentliche Geltung kann somit durch die Gewährung oder Verweigerung von Rechten gefördert oder ausgehöhlt werden.

All dies deutet auf eine dynamische Beziehung zwischen der formellen und der informellen Dimension von Rechten hin. Die Förderung von öffentlichem Verständnis und Sympathie für eine Gruppe kann deren Zugang zu moralischen Ressourcen verbessern, die dann beim Einfordern weiterer Rechte eingesetzt werden können. Während das Einfordern von Rechten in gewissem Sinne einer Beanspruchung gesellschaftlicher Geltung gleichkommt, kann - entsprechend Honneths Argumentation (1995), dass Rechte eine Form der „Anerkennung“ darstellen - die Zuerkennung eines Rechtes das Bild einer Gruppe in der Öffentlichkeit bestärken oder aufbessern. Verschlechtern sich hingegen die öffentliche Geltung oder die moralischen Ressourcen einer Gruppe, so erschwert dies nicht nur die Einforderung von Rechten, sondern kann sogar eine aktive Aushöhlung der jeweiligen Rechte nach sich ziehen. Ebenso kann die öffentliche Geltung einer Gruppe, der Rechte verweigert werden, abnehmen, und diese Möglichkeiten lassen sich im politischen Prozess einsetzen.

Dieser Mechanismus findet sich implizit in der von Schnieder/Ingram (1993) vorgetragenen Argumentation zur sozialen Konstruktion von Zielgruppen im politischen Entscheidungsprozess, wonach das öffentliche Bild einer Gruppe zu politischen Zwecken geformt oder funktionalisiert werden kann. Politiker müssen einerseits ihre Hand- lungen rechtfertigen und sich den Rückhalt der Bevölkerung verschaffen, indem sie demonstrieren, eine Vorstellung vom öffentlichen Wohl zu haben; gleichzeitig müssen sie sich gegenüber dringenden öffentlichen Anliegen aufgeschlossen zeigen. Bevölkerungsgruppen zu Zielgruppen aufzubauen, kann zur Erfüllung beider Zwecke beitragen, indem auf diese Weise ein angebliches öffentliches Problem geschaffen wird und man dann für sich in Anspruch nehmen kann, sich um dessen Lösung zu kümmern. Im Laufe dieses Prozesses reagieren Politiker nach Schnieder/Ingram nicht nur auf das öffentliche Bild bestimmter Zielgruppen, sondern gestalten ihre öffentliche Geltung als „Gewinnler“, „Konkurrenten“, „Abhängige“ (von öffentlichen Leistungen) oder „Abweichler", die durch politische Maßnahmen entsprechend behandelt werden sollten, aktiv mit. Schnieder/Ingram nennen die Zuwanderungspolitik als ein gutes Beispiel für die Funktionsweise dieser sozialen Konstruktion. Verbinden wir den Ansatz dieser Autoren mit einer Analyse bürgerrechtlicher Schichtung, so werden wir aufmerksam auf einen möglichen Zusammenhang zwischen Rechten, Kontrollen und öffentlichem Empfinden, der durch den politischen Prozess selbst erzeugt wird.

Wenngleich die oben skizzierten Beziehungen ein wenig spekulativ sind, sind sie doch empirischer Untersuchung zugänglich, und es lassen sich immerhin einige beeindruckende Beispiele darlegen. Im Folgenden beziehe ich mich auf Großbritannien, dem mir am besten bekannten System, doch würde ich erwarten, dass man andernorts auf Ähnliches stoßen würde.

\subsection{Die politische Debatte zum Thema Asyl und Sozialleistungsansprüche}

Als Reaktion auf steigende Asylbewerberzahlen wurden in Großbritannien im Zeitraum 1996-2002 drei Versuche unternommen, Asylsuchenden, die ihren Asylantrag nicht sofort bei der Einreise gestellt hatten (in country anstelle at port), das Recht auf soziale Unterstützung zu entziehen. Alle drei Versuche wurden letztendlich auf dem Gerichtsweg gekippt, ${ }^{4}$ doch liefert die Tonart der Debatte über die Formulierung dieser politischen Programme ein interessantes Beispiel für die beschriebene Dynamik. In einer Vorankündigung des Vorhabens sagte der damalige Minister für soziale Sicherheit: „The trouble is our system almost invites people to claim asylum, to gain British benefits. Most people who claim asylum don't arrive here as refugees. They come as visitors, tourists or students. And they accept that they should support themselves. The problem is that if they later claim asylum, they can automatically claim benefits. That can't be right. And we're going to stop it." (zitiert in House of Commons 1996, S. 6).

4 Vgl. hierzu ausführlich Morris (2010). 
Diese Argumentation stellt eine Verbindung her zwischen dem Einreisemodus, den Beweggründen für den Asylantrag und der Begründetheit desselben und stempelt in einem Atemzug eine ganze Kategorie von Asylsuchenden $\mathrm{zu}$ „Scheinasylanten“. Auf das so geschaffene Muster von gedanklichen Assoziationen sollte dann reagiert werden durch den Entzug sozialer Unterstützungsleistungen für Asylbewerber, die ihren Antrag nicht sofort bei der Einreise gestellt hatten (damals $70 \%$ aller Asylbewerber), mit folgender Begründung: „They had to demonstrate that they had the means to support themselves when here and the means to return home subsequently[...]. In those circumstances, it seems perfectly reasonable that they should not receive benefit if they subsequently change their story and declare themselves to be asylum seekers." (Hansard [Parlamentsstenogramme] 1995)

Aus der Opposition heraus widersprach die Labour Party dieser - später auf dem Gerichtsweg ausgehebelten Maßnahme, unterbreitete aber nach einiger Zeit an der Regierung mit dem Nationality Immigration and Asylum Act von 2002 einen nahezu identischen Vorschlag mit ähnlicher Begründung.

Aus diesem kurzen Abriss lässt sich eine mögliche Abfolge des Abbaus von Rechten ableiten: Auf die Herausarbeitung eines Problems, zum Beispiel einer hohen Anzahl von Asylanträgen, folgt die Diskreditierung einer breiten Teilgruppe der Asylbewerber, indem man ihr Missbrauch unterstellt, wiederum gefolgt von einer politischen Initiative, die das Problem angeblich lösen soll. Nach Lockwoods Konzept kann die Konsolidierung moralischer und materieller Ressourcen eine Ausweitung von Rechten zur Folge haben; der eben beschriebene Fall deutet auf die Möglichkeit einer umgekehrten Dynamik hin: eine Untergrabung der öffentlichen Geltung bereits im Lande befindlicher Asylantragssteller durch Missbrauchsbezichtigungen, gefolgt von einem Abbau der Rechte dieser Gruppe mit dem Effekt einer weiteren Verfestigung ihres negativen Ansehens.

Die Vermutung einer solchen negativen Wirkung auf die öffentliche Meinung deckt sich mit Untersuchungsergebnissen (McLaren/Johnson 2004), die für den Zeitraum 1995-2003 einen Anstieg einwanderungsfeindlicher Einstellungen unter Labour-Wählern von 58 auf $71 \%$ zeigen und einen generellen Kulturumschwung andeuten, der sich in bis dahin vergleichsweise immigrationsfreundlich gesinnten Kreisen besonders bemerkbar machte. Bekräftigt wird der mögliche Zusammenhang zwischen diesem Stimmungswandel und einer zunehmend aggressiven Einwanderungs- und Asylpolitik auch durch Belege, die die Hohe Flüchtlingskommission der Vereinten Nationen dem Gemeinsamen Menschenrechtsausschuss des britischen Oberund Unterhauses vorlegte: „In the United Kingdom asylum seekers - and the refugees among them - have increasingly become tools for politicians, or have been turned into mere statistics by the popular press. Asylum seekers are easy to demonise. They are foreign, so an attractive target for those who are suspicious of, or actively dislike, foreigners or minorities with foreign origins." (Joint Committee on Human Rights 2007, Ev. 429)

Die Frage des Anspruchs von Asylsuchenden auf Sozialleistungen kann somit als ein Beispiel dienen, wie die Dynamik des Abbaus von Rechten funktioniert.

\subsection{Verwendung des Begriffs „illegal“}

In einem ähnlichen Zusammenhang gab der Beschwerdeausschuss des britischen Presseverbandes (PCC) als Reaktion auf eine steigende Anzahl von Beschwerden über den sprachlichen Umgang mit Flüchtlingen und Asylbewerbern in der britischen Presse im Oktober 2003 einen Leitfaden mit folgendem Wortlaut heraus: ${ }^{5}$ "As an asylum seeker is someone currently seeking refugee status or humanitarian protection, there can be no such thing in law as an 'illegal asylum seeker." Weiterhin heißt es in der Mitteilung, der Verhaltenskodex des Beschwerdeausschusses „has underlined the danger that inaccurate, misleading or distorted reporting may generate an atmosphere of fear and hostility that is not borne out by the facts."

Knapp zehn Jahre nach der Veröffentlichung dieser Aussage flammte diese Debatte jedoch erneut auf, als sich mehrere Organisationen für Migrantenrechte über die Verwendung der Bezeichnung ,illegal migrant“ in der Tageszeitung The Guardian beschwerten. Diese bat ihre Leser um Kommentare und um Unterstützung bei der Wahl einer besseren Formulierung, was die Organisation Migrant Rights Network zu folgender Antwort veranlasste: „Deeming a person an 'illegal migrant' is useful because it permits the state to act against individuals in circumstances when their actions are normal, mundane and do not give rise to any obvious socially harmful effects. The archetypical 'illegal' migrant is the 'illegal migrant worker'[...] (whose) work ceases to be positive and socially valuable and is transformed into a threat. ${ }^{\text {"6 }}$ Auch dieser Kommentar zeigt, wie öffentliche Ressentiments geschürt werden können, um den Weg für aggressivere Kontrollen freizumachen.

\subsection{Freizügigkeit für rumänische und bulgarische Bürger}

Als drittes Beispiel ließe sich die hitzige Debatte über die Aufhebung der Beschränkungen der Arbeitnehmerfreizügigkeit für bulgarische und rumänische Arbeitskräfte ab Januar 2014 heranziehen, die im EU-Recht festgelegt ist. Im Zuge der Vorbereitungen auf die Änderung und im Zusammenhang mit übertrieben hohen Schätzungen des zu erwartenden Zuzugs aus den genannten Ländern

5 http://www.pcc.org.uk (letzter Zugriff: 06.10.14).

6 http://www.migrantsrights.org.uk/ (letzter Zugriff: 08.09.14). 
(Guardian 2013b) beeilte sich die britische Regierung, Rechtsänderungen durchzusetzen, die den Anspruch auf staatliche Unterstützung und öffentliche Dienstleistungen einschränken. Der Kommissar für Menschenrechte des Europarats beklagte die damit verbundene „beschämende Rhetorik“, die rumänische und bulgarische Migranten aufs Korn nehme und drohe, Vorurteile und Feindseligkeit zu verstärken. „A stigma is put on Bulgarian and Romanian citizens just because of their origin. This is unacceptable because a state cannot treat Bulgarian and Romanian citizens differently from other EU citizens. They need to be treated as everyone else, not on the basis of assumptions or generalisations about their ethnic origin." (Guardian 2013a).

Seine Aufforderung an die politische Führung Großbritanniens, sich aktiver für eine Entschärfung der Einwanderungsdebatte einzusetzen und den positiven Beitrag der Wanderarbeitnehmer herauszustellen, kann als weiterer Hinweis auf eine mögliche Verbindung von politischen Absichtserklärungen und öffentlicher Meinung gedeutet werden.

Zwar liefert keines der drei angeführten Beispiele eindeutige Beweise für einen Zusammenhang zwischen politischer Rhetorik, Einwanderungspolitik und öffentlicher Stimmung, doch deuten sie alle auf den weiter oben beschriebenen zirkulären Prozess hin. Danach setzt die Dynamik des Abbaus von Rechten ein, sobald eine Schmälerung der öffentlichen Geltung einer Bevölkerungsgruppe eine Einschränkung von deren Rechten rechtfertigt, welche wiederum das negative Image der Betroffenen konsolidiert und deren moralische Ressourcen weiter untergräbt.

\section{Schlussfolgerung}

Ich habe in diesem Aufsatz argumentiert, dass mit Hilfe des Konzeptes der bürgerrechtlichen Schichtung ein Mittelweg zwischen zwei Argumentationen beschritten werden kann, die allzu optimistisch von postnationaler Expansion von Bürgerrechten beziehungsweise allzu restriktiv von nationaler Abschottung sprechen. Dadurch eröffnet sich der Forschung ein breiteres Feld, in welchem es um die Funktion von Rechten in der Gegenwartsgesellschaft geht, als Instrument der Governance und als dynamischer Raum für gesellschaftliche Auseinandersetzung und Aushandlung. Aus der Perspektive der formellen Dimension bürgerrechtlicher Schichtung lassen sich die Wege erhellen, mit denen die Einwanderungsgesetzgebung ein strukturiertes System der Ungleichheit bezüglich von Rechten schafft, während die informelle Dimension großes Potenzial zur Erkenntnis des dynamischen Wesens dieses Systems hat. In Weiterführung von Lockwoods Konzept habe ich ausgeführt, dass moralische Ressourcen und gesellschaftliche Geltung beim Aufbau legitimer Ansprüche auf Rechte eine wichtige Rolle spielen, gleichzeitig aber von Aushöhlung bedroht sind - besonders im Zusammenhang mit dem meinungsbildenden Einfluss politischer Rhetorik und politischer Maßnahmen. Während der letzte Teil meines Papers diese Problematik als vernachlässigten Aspekt von Lockwoods Modell in den Blick nimmt, darf die Rolle von Rechten als Mittel zur Stärkung benachteiligter Gesellschaftsgruppen ebenso wenig vernachlässigt werden wie ihre Bedeutung für das, was Alexander (2006) als „civil repair“ bezeichnet, denn Rechte wirken nicht nur als soziale Konstrukte, sondern auch als soziale Kraft, und sie sind folglich immer umstritten.

\section{LITERATUR}

Alexander, J. C. (2006): The civil sphere, Oxford

Arendt, H. (1979[1948]): The origins of totalitarianism, New York

Benhabib, S. (2004): The rights of others, Cambridge

Bobbio, N. (1995): The age of rights, Cambridge

Brubaker, W. R. (Hrsg.) (1989): Immigration and the politics of citizenship in Europe and America, Lanham MD

Castles, S./Miller, M. (1993): The age of migration, Houndsmill

Crawley, H. (1997): Women as asylum seekers, Immigration Law Practitioners Association, London

Cruz, A. (1995): Shifting responsibility, Stoke-on-Trent

European Migration Network (2013): Intra-EU mobility of third country nationals, European Commission, Brussels

Foucault, M. (1991): Governmentality, in: Burchell, G./Gordon, P./Miller, P. (Hrsg.): The Foucault Effect, Hemel Hempstead, S. 85-104

Guardian (2013a): European watchdog accuses Britain of shameful rhetoric on migrants

Guardian (2013b): Bulgaria issues fierce rebuke to David Cameron over migrants Guardian (2014): PM tries to placate right with new curb on EU migrants benefits Hammar, T. (1990): Democracy and the nation State, Aldershot

Hansard [Parlamentsstenogramme] (1995/1996): HC Debate, 23rd Jan, col 137 Honneth, A. (1995): The struggle for recognition, Cambridge

House of Commons (1996): Social security changes for asylum seekers and other persons from abroad, Research Paper 96/9

Joint Committee on Human Rights (2007): Tenth Report, London

Joppke, C. (1999): Immigration and the nation state, Oxford

Kofman, E./Phizacklea, A./Raghuram, P./Sales, R. (2000): Gender and international migration in Europe. Employment, welfare and politics, London/New York Lockwood, D. (1996): Civic integration and class formation, in: British Journal of Sociology 47 (3), S. $531-550$

McLaren, L./Johnson, M. (2004): Understanding the rising tide of anti-immigrant sentiment, in: Park, A./Curtice, J./Thompson, K./Bromley, C./Phillips, M (Hrsg.): British Social Attitudes, 21st Report, S. 169-200

Morissens, A./Sainsbury, D. (2005): Migrants' social rights, ethnicity and welfare, in: Journal of Social Policy 34 (4), S.637-660

Morris, L. D. (2002): Managing migration, London

Morris, L. D. (2003): Managing contradiction, in: International Migration Review 37 (1), S. 74-100

Morris, L. D. (2010): Asylum, welfare and the cosmopolitan ideal, London Sassen, S. (1998): Globalisation and its discontents, New York

Schnieder, A./Ingram, H. (1993): Social construction of target populations, in: American Political Science Review 87 (2), S. 334-346

Soysal, Y. (1994): Limits of citizenship, Chicago

\section{AUTORIN}

LYDIA MORRIS, PhD, ist Professorin für Soziologie und Research Director am Department of Sociology, University of Essex. Arbeitsschwerpunkte: Migrationspolitik in der EU und Menschenrechtsfragen, Armut und Sozialpolitik.

Idmorris@essex.ac.uk 